\title{
Fracture of Circular Pipes by Internal Pressure
}

\author{
T. Y. THOMAS
}

\section{Contents}

1. Introduction . . . . . . . . . . . . . . . . 623

2. General symmetry and equilibrium conditions . . . . . . 624

3. Elastic deformation of the pipe . . . . . . . . . . . . . 626

4. Yield condition. Beginning of plastic deformation . . . . . 629

5. Plastic deformation of the pipe. Boundary conditions . . . 630

6. Solution of the plastic equilibrium problem . . . . . . . . 631

7. Justification of the tentative yield condition . . . . . . . 634

8. Surface of strong instability. Plastic flow . . . . . . . . 635

9. Violation of circular symmetry . . . . . . . . . . . 636

10. Fracture in pipes . . . . . . . . . . . . . 638

1. Introduction. The occurrence of a small slip of the material particles or discontinuity in the particle velocity over a surface in a solid will, in general, be damped out as a consequence of the boundary conditions and the conditions imposed by the equations governing the behavior of the material. For certain special surfaces, however, the discontinuity will become indefinitely large within a finite time. These latter surfaces, called surfaces of strong instability, have been proposed as surfaces over which the solid will fracture or rupture. On the basis of this criterion we have shown in several previous publications ${ }^{1}$ that a plate under uniform tension at the yield point may fracture along the well known Lüders band and correspondingly that a circular bar, stressed uniformly in the direction of its axis, is subject to fracture over a plane perpendicular to the axis or along a circular cone whose axis coincides with the axis of the bar.

This article ${ }^{2}$ deals with the fracture of a circular pipe produced solely by

1See T. Y. Thomas, Plastic flow and fracture in solids, Jour. of Math. \& Mech. 7 (1958), pp. 291-322, also Rupture of round bars by tension, ibid., 8 (1959), pp. 1-25.

2This investigation was conducted at the request of the Naval Research Laboratory and has been supported by the Office of Naval Research under Contract Nonr-908(09), Indiana University, NR 041037. 
uniform internal pressure perpendicular to its central axis. We have included an expository section on the purely elastic situation which arises when the internal pressure is not sufficiently large to produce the plastic strains necessary for fracture; this leads to the value of the pressure $p$ just sufficient to initiate plastic deformation on the inner surface of the pipe (see §3). In this discussion, as well as in our subsequent discussion of the fracture of the fully plastic pipe, we have followed $\mathrm{HILL}^{3}$ in assuming the Tresca yield condition, which has the advantage over the von Mises or quadratic yield condition in that it permits us to express the radial and angular components of the stress in the plastically deformed pipe by means of simple elementary functions. By using the von Mises stress-strain relations rather than relations of Prandtl-Reuss type, as we have done in our discussion of this problem, the longitudinal or axial component of the stress can be expressed in a similar manner. The remaining components of the stress tensor vanish in consequence of the boundary conditions and the conditions imposed by cylindrical symmetry. Finally we have given a complete analysis of the plastic displacements arising in this problem.

Let $\Sigma$ be the intersection of the pipe, assumed to be entirely in a state of plastic equilibrium, with a half plane through its central axis. We have shown in $\$ 10$ that the plane $\Sigma$ is a surface of strong instability in the plastically deformed pipe. This result, namely that fracture may occur in the pipe over the plane $\Sigma$, is confirmed by laboratory tests. ${ }^{4} \mathrm{~A}$ formula for the fracture pressure $p$ is given in $\$ 6$.

2. General symmetry and equilibrium conditions. Let the central axis of the pipe be the $z$ axis of a system of cylindrical coordinates and denote by $r$ and $\theta$ the radial and angular coordinates of this system. The cylindrical coordinates $r, \theta$ and $z$ will also be denoted by $x^{1}, x^{2}$ and $x^{3}$ respectively. This latter notation permits us to identify the components of the particle displacement $u$ and the components of the stress tensor $\sigma$ with the coordinate directions. Thus the quantities $u_{1}, u_{2}$ and $u_{3}$ will represent the covariant radial, angular and longitudinal components of the particle displacement.

In the cylindrical coordinate system the covariant components $g_{i j}$ of the metric tensor are given by the matrix

$$
\left\|\begin{array}{lll}
g_{11} & g_{12} & g_{13} \\
g_{21} & g_{22} & g_{23} \\
g_{31} & g_{32} & g_{33}
\end{array}\right\|=\left\|\begin{array}{lll}
1 & 0 & 0 \\
0 & r^{2} & 0 \\
0 & 0 & 1
\end{array}\right\| .
$$

Correspondingly the contravariant components of this tensor are given by

${ }^{3}$ R. Hulu, The Mathematical Theory of Plasticity, Oxford, Clarendon Press, 1950, p. 111. 259.

${ }^{4}$ See A. NadaI, Theory of Flow and Fracture in Solids, McGraw-Hill, New York, 1950, p. 


$$
\left\|\begin{array}{lll}
g^{11} & g^{12} & g^{13} \\
g^{21} & g^{22} & g^{23} \\
g^{31} & g^{32} & g^{33}
\end{array}\right\|=\left\|\begin{array}{ccc}
1 & 0 & 0 \\
0 & 1 / r^{2} & 0 \\
0 & 0 & 1
\end{array}\right\| .
$$

Hence from the relations between the covariant and contravariant components of the displacement $u$, namely

we see that

$$
u_{i}=g_{i j} u^{i}
$$

$$
u_{1}=u^{1} ; \quad u_{2}=r^{2} u^{2} ; \quad u_{3}=u^{3} .
$$

The natural symmetry conditions of our problem can be expressed most conveniently when use is made of the above cylindrical coordinates. Relative to the cylindrical system it is obvious, from symmetry, that the components $u_{i}$ will be independent of the angular coordinate $\theta$. Also the angular component $u_{2}$ must vanish and the radial component $u_{1}$ must be independent of the $z$ coordinate. Hence (2.1) becomes

$$
u_{1}=u^{1} ; \quad u_{2}=u^{2}=0 ; \quad u_{3}=u^{3},
$$

and there is therefore no distinction between the values of the covariant and contravariant components of the displacement. Moreover, it is clear from symmetry that a plane section in the pipe, perpendicular to the central or $z$ axis, must be displaced into a plane section perpendicular to this axis. Hence $u_{3}$ must be independent of the coordinate $r$. In the case of the equilibrium problem, for which the quantities $u_{i}$ are independent of the time, we can summarize these symmetry requirements by writing

$$
u_{1}=u_{1}(r) ; \quad u_{2}=0 ; \quad u_{3}=u_{3}(z) .
$$

Similarly we see that the stress components $\sigma_{i}$ must be independent of the $\theta$ and $z$ coordinates. In addition it follows from symmetry that the two components $\sigma_{12}$ and $\sigma_{23}$ must vanish in the cylindrical system. ${ }^{5}$ Corresponding to (2.2) we can therefore write

$$
\sigma_{12}=\sigma_{23}=0 ; \quad \sigma_{i i}=\sigma_{i j}(r) \quad \text { otherwise. }
$$

It will be helpful in the following discussion to have the explicit expressions for the covariant derivatives of the displacement $u$ and the stress tensor $\sigma$ in the cylindrical system. These derivatives are given by the formulæ

$$
\begin{gathered}
u_{i, i}=\frac{\partial u_{i}}{\partial x^{j}}-u_{m} \Gamma_{i i}^{m}, \\
\sigma_{i j, k}=\frac{\partial \sigma_{i j}}{\partial x^{k}}-\sigma_{m i} \Gamma_{i k}^{m}-\sigma_{i m} \Gamma_{i k}^{m},
\end{gathered}
$$

${ }^{5}$ Cp. S. Trmoshenko, Theory of Elasticity, McGraw-Hill, New York, 1934, p. 309. 
where the $\Gamma$ 's are the components of the affine connection. In general

$$
\Gamma_{j k}^{i}=\frac{1}{2} g^{i m}\left(\frac{\partial g_{m i}}{\partial x^{k}}+\frac{\partial g_{m k}}{\partial x^{j}}-\frac{\partial g_{j k}}{\partial x^{m}}\right) .
$$

Hence, using the values of the $g$ 's given by the above matrices, we find that

$$
\Gamma_{22}^{1}=-r ; \quad \Gamma_{12}^{2}=\frac{1}{r} ; \quad \Gamma_{j k}^{i}=0 \quad \text { otherwise. }
$$

Making use of (2.2), (2.3) and (2.6) it then follows from (2.4) and (2.5) that

$$
\left\{\begin{array}{lll}
u_{1,1}=\frac{\partial u_{1}}{\partial r} ; & u_{1,2}=0 ; & u_{1,3}=0 \\
u_{2,1}=0 ; & u_{2,2}=r u_{1} ; & u_{2,3}=0 \\
u_{3,1}=0 ; & u_{3,2}=0 ; & u_{3,3}=\frac{\partial u_{3}}{\partial z}
\end{array}\right.
$$

and

$$
\left\{\begin{array}{lll}
\sigma_{11,1}=\frac{\partial \sigma_{11}}{\partial r} ; & \sigma_{12,2}=r \sigma_{11}-\frac{\sigma_{22}}{r} ; & \sigma_{13,3}=0 \\
\sigma_{21,1}=0 ; & \sigma_{22,2}=0 ; & \sigma_{23,3}=0 \\
\sigma_{31,1}=\frac{\partial \sigma_{13}}{\partial r} ; & \sigma_{32,2}=r \sigma_{13} ; & \sigma_{33,3}=0 .
\end{array}\right.
$$

Now consider the equilibrium equations

$$
g^{i k} \sigma_{i i, k}=0 .
$$

For $i=2$ we see immediately from (2.8) that (2.9) is satisfied identically. However, for $i=1$ and $i=3$ the equations (2.9) yield

$$
\frac{\partial \sigma_{11}}{\partial r}+\frac{\sigma_{11}}{r}-\frac{\sigma_{22}}{r^{3}}=0 ; \quad \frac{\partial \sigma_{13}}{\partial r}+\frac{\sigma_{13}}{r}=0 .
$$

Similarly, using (2.7), we can readily calculate the divergence of the displacement $u$. Thus we find that

$$
g^{i i} u_{i, j}=\frac{\partial u_{1}}{\partial r}+\frac{u_{1}}{r}+\frac{\partial u_{3}}{\partial z} .
$$

3. Elastic deformation of the pipe. Suppose the (constant) internal pressure $p$ is insufficient to cause plastic deformation within the pipe. Between the displacement $u$ and the stress $\sigma$ we shall then have the elastic stress-strain relations, namely

$$
\sigma_{i j}=\lambda\left(g^{a b} u_{a, b}\right) g_{i i}+\mu\left(u_{i, i}+u_{i, i}\right),
$$

in which $\lambda$ and $\mu$ are the usual elastic constants. To deduce the explicit conse- 
quences of these relations we must assign specific values to the indices $i, j$ and then make use of the equations, such as (2.7) and (2.11), which we derived in $\$ 2$.

Taking $i, j=1,2$ and $i, j=2,3$ we find that (3.1) is satisfied identically. For $i, j=1,3$ the relation becomes

$$
\sigma_{13}=0 .
$$

Hence the second equilibrium condition (2.10) is satisfied automatically and there is a slight simplification in the last row of the set of equations (2.8).

The remaining conditions resulting from (3.1) are obtained by taking

$$
i, j=1,1 ; \quad i, j=2,2 ; \quad i, j=3,3 .
$$

Making these substitutions in turn we find that

$$
\begin{aligned}
& \sigma_{11}=(\lambda+2 \mu) \frac{\partial u_{1}}{\partial r}+\lambda\left(\frac{u_{1}}{r}+\frac{\partial u_{3}}{\partial z}\right), \\
& \frac{\sigma_{22}}{r^{2}}=\lambda\left(\frac{\partial u_{1}}{\partial r}+\frac{\partial u_{3}}{\partial z}\right)+(\lambda+2 \mu) \frac{u_{1}}{r}, \\
& \sigma_{33}=\lambda\left(\frac{\partial u_{1}}{\partial r}+\frac{u_{1}}{r}\right)+(\lambda+2 \mu) \frac{\partial u_{3}}{\partial z} .
\end{aligned}
$$

From (2.2), (2.3) and (3.3) we see that the derivative $\partial u_{3} / \partial z$ must be a constant. Hence, since $u_{3}$ can depend at most on the variable $z$, we must have

$$
u_{3}=C z+D \text {, }
$$

where $C$ and $D$ are constants. Substituting the values of $\sigma_{11}$ and $\sigma_{22}$ given by (3.3) and (3.4) into the first relation (2.10) we find, after some reduction of the resulting equation, that

$$
\frac{d^{2} u_{1}}{d r^{2}}+\frac{1}{r} \frac{d u_{1}}{d r}-\frac{u_{1}}{r^{2}}=0
$$

where we have now used the symbol of ordinary differentiation since $u_{1}$ can depend only on the coordinate $r$ from (2.2). Integrating (3.7) we have

$$
u_{1}=A r+\frac{B}{r},
$$

where $A$ and $B$ are constants. Hence from (3.6) and (3.8) we find that (3.3), (3.4) and (3.5) can be written

$$
\begin{aligned}
& \sigma_{11}=2(\lambda+\mu) A-2 \mu \frac{B}{r^{2}}+\lambda C, \\
& \frac{\sigma_{22}}{r^{2}}=2(\lambda+\mu) A+2 \mu \frac{B}{r^{2}}+\lambda C, \\
& \sigma_{33}=2 \lambda A+(\lambda+2 \mu) C .
\end{aligned}
$$


Denoting by $a$ the inner radius and by $b$ the outer radius of the pipe in its strained position we have

$$
\begin{array}{lll}
\sigma_{11}=-p & \text { for } & r=a, \\
\sigma_{11}=0 & \text { for } & r=b,
\end{array}
$$

where $p$ is the internal pressure. Hence from (3.9) we obtain

$$
\begin{aligned}
& 2(\lambda+\mu) A-2 \mu \frac{B}{a^{2}}+\lambda C=-p, \\
& 2(\lambda+\mu) A-2 \mu \frac{B}{b^{2}}+\lambda C=0 .
\end{aligned}
$$

Subtracting corresponding members of (3.14) and (3.15) we find that

$$
B=\frac{a^{2} b^{2} p}{2 \mu\left(b^{2}-a^{2}\right)} \cdot
$$

A condition involving $A$ and $C$ alone is obtained from (3.14) or (3.15) by elimination of $B$ by means of the substitution (3.16). Thus

$$
2(\lambda+\mu) A+\lambda C=\frac{a^{2} p}{b^{2}-a^{2}} .
$$

Another equation in $A$ and $C$ is obtained from the fact that no load is applied on the ends of the pipe (see $\$ 1$ ). Since the ends of the pipe are planes perpendicular to the central axis, i.e. cross sections, and since $\sigma_{33}$ is a constant according to (3.11), it follows that the load is given by $\sigma_{33} S$ where $S$ is the area of a cross section of the pipe. Hence $\sigma_{33}=0$ and hence from (3.11) we have

$$
2 \lambda A+(\lambda+2 \mu) C=0 .
$$

Solving (3.17) and (3.18) for $A$ and $C$ we now find that

$$
\begin{aligned}
& A=\frac{(\lambda+2 \mu) a^{2} p}{2 \mu(3 \lambda+2 \mu)\left(b^{2}-a^{2}\right)}, \\
& C=\frac{-\lambda a^{2} p}{\mu(3 \lambda+2 \mu)\left(b^{2}-a^{2}\right)} .
\end{aligned}
$$

It is seen from the above equation (3.20) that the constant $C$ must be different from zero. Hence the constant $D$ in (3.6) can be reduced to zero by a proper choice of the origin of our coordinate system and we can therefore write

$$
u_{3}=C z
$$

in place of (3.6). Hence, since $u_{2}=0$, the equations (3.8) and (3.21) in which $A, B$ and $C$ are given by (3.19), (3.16) and (3.20) respectively will determine the particle displacements. Moreover, elimination of the constants $A, B$ and $C$ from (3.9) and (3.10) by means of the substitutions (3.19), (3.16) and (3.20) 
provides us with the determination of the components $\sigma_{11}$ and $\sigma_{22}$ of the stress tensor, and since the remaining components of this tensor have been shown to vanish we thus arrive at the complete solution of the purely elastic problem.

For reference in the following discussion we observe that the above elimination of the constants $A, B$ and $C$ from (3.9) and (3.10) leads to the following relations:

$$
\begin{aligned}
\sigma_{11} & =\left(1-\frac{b^{2}}{r^{2}}\right) \frac{a^{2} p}{b^{2}-a^{2}}, \\
\frac{\sigma_{22}}{r^{2}} & =\left(1+\frac{b^{2}}{r^{2}}\right) \frac{a^{2} p}{b^{2}-a^{2}} .
\end{aligned}
$$

4. Yield condition. Beginning of plastic deformation. The principal stresses $\sigma_{1}, \sigma_{2}$ and $\sigma_{3}$ are given as the three solutions $\sigma_{k}$ of the determinantal equation

$$
\left|\sigma_{i j}-\sigma_{k} g_{i j}\right|=0 \text {. }
$$

For the case of the elastically deformed pipe considered in $\S 3$ the equation (4.1) becomes

$$
\left|\begin{array}{ccc}
\sigma_{11}-\sigma_{k} & 0 & 0 \\
0 & \sigma_{22}-\sigma_{k} r^{2} & 0 \\
0 & 0 & -\sigma_{k}
\end{array}\right|=0,
$$

since all components $\sigma_{i j}$ vanish with the exception of $\sigma_{11}$ and $\sigma_{22}$ which are given by the equations (3.22) and (3.23). Selecting the principal values $\sigma_{1}$, $\sigma_{2}$ and $\sigma_{3}$ so that

$$
\sigma_{3} \geqq \sigma_{2} \geqq \sigma_{1},
$$

we see immediately from (4.2) and the two equations (3.22) and (3.23) that

$$
\sigma_{3}=\left(1+\frac{b^{2}}{r^{2}}\right) \frac{a^{2} p}{b^{2}-a^{2}} ; \quad \sigma_{2}=0 ; \quad \sigma_{1}=\left(1-\frac{b^{2}}{r^{2}}\right) \frac{a^{2} p}{b^{2}-a^{2}} .
$$

In this paper we shall assume the Tresca yield condition in accordance with which yield may occur whenever the equation

$$
\sigma_{3}-\sigma_{1}=J
$$

is satisfied, where $J$ is a material constant for the solid. It is considered here that the inequality (4.3) holds so that the left member of (4.5) represents the difference between the greatest and least of the principal values of the stress. Making the substitution (4.4) in (4.5) we obtain

$$
\frac{2 a^{2} b^{2} p}{\left(b^{2}-a^{2}\right) r^{2}}=J
$$

as the explicit form of the yield condition for the circular pipe under elastic deformation. 
If the internal pressure $p$ is continually increased from a value for which the pipe is entirely in a state of elastic equilibrium, we see immediately from (4.6) that yield will begin on the inner surface of the pipe, i.e. for $r=a$. Hence we can state the following result. Yield will first occur on the inner surface of the pipe for a value of the internal pressure given by

$$
p=\frac{1}{2}\left(1-\frac{a^{2}}{b^{2}}\right) J .
$$

5. Plastic deformation of the pipe. Boundary conditions. Suppose the internal pressure $p$ is allowed to increase to such an extent beyond the value given by (4.7) that the pipe will enter a state of complete plastic equilibrium. In treating this problem we shall use the previous symbols $u_{i}$ and $\sigma_{i j}$ to denote the displacements and the components of the stress tensor in the plastically deformed pipe. It is assumed that the plastic displacements $u_{i}$ and the stress components $\sigma_{i j}$ satisfy the general symmetry and equilibrium conditions in $\$ 2$.

We now assume the validity of the following relations:

$$
\begin{aligned}
g^{i j} u_{i, i} & =0, \text { equation of incompressibility, } \\
\boldsymbol{\epsilon}_{i j} & =\psi \sigma_{i j}^{*}, \text { stress-strain equations, }
\end{aligned}
$$

where $\psi$ is a positive factor of proportionality, the $\epsilon_{i j}$ are the components of the strain tensor and the $\sigma_{i i}^{*}$ are the components of the stress deviator. These tensors are defined explicitly by the equations

$$
\epsilon_{i j}=\frac{1}{2}\left(u_{i, i}+u_{j, i}\right) ; \quad \sigma_{i j}^{*}=\sigma_{i j}-\frac{1}{3}\left(g^{a b} \sigma_{a b}\right) g_{i j} .
$$

In addition we assume the Tresca condition (4.5) in which $\sigma_{3}$ and $\sigma_{1}$ are the greatest and least of the principal values of the stress tensor.

As stated in $\$ 2$ it follows from symmetry that a plane section of the pipe, perpendicular to its central axis, must be displaced into a plane section which is likewise perpendicular to this axis. In particular, the flat ends of the pipe will remain flat, i.e. perpendicular to the central axis of the pipe. Hence, since the components $\sigma_{i j}$ depend at most on the coordinate $r$ by (2.3), the condition that there is no load on the ends of the pipe can be expressed as

$$
\iint \sigma_{33} d S=0
$$

where the integration is over a plane section of the pipe perpendicular to the central axis. Or, in place of (5.3), we can write more simply

$$
\int_{a}^{b} r \sigma_{33} d r=0
$$

where $a$ and $b$ are the inner and outer radii of the pipe in its strained position. Beyond this condition there are the boundary conditions given by (3.12) and (3.13) which will enter into the discussion of this problem. 
6. Solution of the plastic equilibrium problem. Using (2.7) and the definition of the quantities $\epsilon_{i j}$ in $\$ 5$ we can write

$$
\left\{\begin{array}{lll}
\epsilon_{11}=\frac{d u_{1}}{d r} ; & \epsilon_{12}=0 ; & \epsilon_{13}=0, \\
\epsilon_{22}=r u_{1} ; & \epsilon_{23}=0 ; & \epsilon_{33}=\frac{d u_{3}}{d z} .
\end{array}\right.
$$

Similarly from (2.3) and the definition of the $\sigma_{i j}^{*}$ we have

$$
\left\{\begin{array}{l}
\sigma_{11}^{*}=\frac{2}{3} \sigma_{11}-\frac{1}{3} \frac{\sigma_{22}}{r^{2}}-\frac{1}{3} \sigma_{33}, \\
\frac{\sigma_{22}^{*}}{r^{2}}=\frac{2}{3} \frac{\sigma_{22}}{r^{2}}-\frac{1}{3} \sigma_{11}-\frac{1}{3} \sigma_{33}, \\
\sigma_{33}^{*}=\frac{2}{3} \sigma_{33}-\frac{1}{3} \frac{\sigma_{22}}{r^{2}}-\frac{1}{3} \sigma_{11}, \\
\sigma_{12}^{*}=0 ; \quad \sigma_{13}^{*}=\sigma_{13} ; \quad \sigma_{23}^{*}=0 .
\end{array}\right.
$$

The condition (5.1) can be written

$$
\frac{d u_{1}}{d r}+\frac{u_{1}}{r}+\frac{d u_{3}}{d z}=0
$$

on account of (2.11). But the last term in the left member of this equation can depend at most on the coordinate $z$ while the first two terms depend at most on the coordinate $r$; hence (6.3) must decompose into the following two relations:

$$
\frac{d u_{1}}{d r}+\frac{u_{1}}{r}=2 M ; \quad \frac{d u_{3}}{d z}=-2 M
$$

where $M$ is a constant. Integrating (6.4) we obtain

$$
u_{1}=M r+\frac{N}{r} ; \quad u_{3}=-2 M z
$$

in which $N$ is an additional constant. The constant of integration in the second relation (6.5) has been taken equal to zero since this is possible by a proper choice of the origin of the cylindrical coordinate system as was shown in $\$ 3$.

For $i, j=1,2$ and $i, j=2,3$ the equations (5.2) are seen to be satisfied identically. However, for $i, j=1,3$ we find that (5.2) yields

$$
\sigma_{13}=0 \text {. }
$$

If we multiply (5.2) by $g^{i j}$ and sum on the repeated indices the resulting equation is satisfied identically on account of (5.1). Hence we need consider only the equations obtained by taking $i, j=1,1$ and $i, j=2,2$ in (5.2). But, when we take account of (6.5) as well as (6.1) and (6.2), we then have 


$$
\begin{aligned}
& M-\frac{N}{r^{2}}=\psi\left(\frac{2}{3} \sigma_{11}-\frac{1}{3} \frac{\sigma_{22}}{r^{2}}-\frac{1}{3} \sigma_{33},\right) \\
& M+\frac{N}{r^{2}}=\psi\left(\frac{2}{3} \frac{\sigma_{22}}{r^{2}}-\frac{1}{3} \sigma_{11}-\frac{1}{3} \sigma_{33}\right) .
\end{aligned}
$$

Subtracting corresponding members of (6.7) and (6.8) we obtain

$$
\frac{2 N}{r^{2}}=\psi\left(\frac{\sigma_{22}}{r^{2}}-\sigma_{11}\right) \text {. }
$$

Let us now assume that the Tresca condition (4.5), which by hypothesis is satisfied in the plastically deformed pipe, can be represented by writing

$$
\frac{\sigma_{22}}{r^{2}}-\sigma_{11}=J
$$

After the stress components $\sigma_{i i}$ have been determined it will be verified that (6.10) is equivalent to the condition (4.5) in which $\sigma_{3}$ and $\sigma_{1}$ are the greatest and least principal values of the stress (see $\S 7$ ). Hence from (6.9) and (6.10) we have

$$
\psi=\frac{2 N}{J r^{2}}
$$

It follows from this relation that the constant $N$ is positive since $\psi$ is positive by hypothesis.

Expanding the equilibrium equations (2.9) we obtain

$$
\begin{aligned}
& \sigma_{11,1}+\frac{1}{r^{2}} \sigma_{12,2}+\sigma_{13,3}=0, \\
& \sigma_{21,1}+\frac{1}{r^{2}} \sigma_{22,2}+\sigma_{23,3}=0, \\
& \sigma_{31,1}+\frac{1}{r^{2}} \sigma_{32,2}+\sigma_{33,3}=0 .
\end{aligned}
$$

But from (2.8) and (6.6) we observe that (6.13) and (6.14) are satisfied identically. However, the equation (6.12) gives us

$$
\frac{d \sigma_{11}}{d r}=\frac{1}{r}\left(\frac{\sigma_{22}}{r^{2}}-\sigma_{11}\right) .
$$

Or, in view of (6.10), we have

$$
\frac{d \sigma_{11}}{d r}=\frac{J}{r}
$$

Integrating (6.15) we obtain

$$
\sigma_{11}=J \log r+\text { const. }
$$


To eliminate the constant in this relation we need only avail ourselves of the boundary condition (3.13). We thus find

$$
\sigma_{11}=J \log \frac{r}{b}
$$

Then, combining this relation with (6.10), we have

$$
\frac{\sigma_{22}}{r^{2}}=J\left(1+\log \frac{r}{b}\right)
$$

Moreover, we obtain the relation

$$
\sigma_{33}=J\left(\log \frac{r}{b}-\frac{3 M}{2 N} r^{2}+\frac{1}{2}\right)
$$

when we eliminate the quantity $\psi$ from (6.7) by the substitution (6.11) and the stress components $\sigma_{11}$ and $\sigma_{22}$ by the substitutions (6.16) and (6.17). Equations (6.16), (6.17) and (6.18) provide us with a determination of the components $\sigma_{11}, \sigma_{22}$ and $\sigma_{33}$ of the stress tensor. The remaining components $\sigma_{12}, \sigma_{13}$ and $\sigma_{23}$ of this tensor vanish on account of (2.3) and (6.6).

Utilization of the boundary condition (3.12) leads to a relation for the internal pressure $p$ in the plastically deformed pipe. Thus from (3.12) and (6.16) we obtain

$$
p=J \log \frac{b}{a}
$$

All equations and conditions stated in $\$ 5$ have now been applied with the exception of the boundary condition (5.4). But this condition becomes

$$
\int_{a}^{b}\left(\log \frac{r}{b}-\frac{3 M}{2 N} r^{2}+\frac{1}{2}\right) r d r=0
$$

on account of (6.18). Performing the various integrations indicated in (6.20) and reducing the resulting equation we obtain the following relation:

$$
a^{2} \log \frac{b}{a}=\frac{3 M}{4 N}\left(b^{4}-a^{4}\right)
$$

Assuming the plastic displacements which enter into this problem to be sufficiently small for us to identify the radii $a$ and $b$ in the above relations with the known values of the corresponding radii of the unstrained pipe, we see that the ratio $M / N$ is determined by (6.21). Hence equations (6.16), (6.17) and (6.18) together with (2.3) and (6.6) provide a complete determination of the stress in the pipe. However, only the ratio of the plastic displacements $u_{1}$ and $u_{3}$ is determined by (6.5). The failure to determine such displacements uniquely is one of the characteristic features of this theory of the plastic behavior of solids.

We observed above that the constant $N$ is positive. Hence, since $b>a$, it is seen from (6.21) that the constant $M$ is also positive. In view of the second equation 
(6.5) this fact permits us to state the following result. There is a contraction of the plastic material in the pipe in the direction of its central axis. This result will have an important bearing in our discussion of the fracture problem in $\$ 10$.

7. Justification of the tentative yield condition. We shall now show that the relation (6.10) is definitely equivalent to the Tresca yield condition (4.5) as was tentatively assumed in the foregoing section. First observe that the general equation (4.1) for the determination of the three principal stresses $\sigma_{k}$ becomes

$$
\left|\begin{array}{ccc}
\sigma_{11}-\sigma_{k} & 0 & 0 \\
0 & \sigma_{22}-\sigma_{k} r^{2} & 0 \\
0 & 0 & \sigma_{33}-\sigma_{k}
\end{array}\right|=0
$$

when the components $\sigma_{i j}$ have the values found in $\S 6$. Hence the three principal stresses $\sigma_{k}$ will be given by the quantities

$$
\sigma_{11} ; \quad \frac{\sigma_{22}}{r^{2}} ; \quad \sigma_{33} .
$$

But it is obvious that if $(6.10)$ is to be a consequence of (4.5) we must take

$$
\sigma_{3}=\frac{\sigma_{22}}{r^{2}} ; \quad \sigma_{2}=\sigma_{33} ; \quad \sigma_{1}=\sigma_{11} .
$$

Our problem is therefore to show that if the principal stresses $\sigma_{1}, \sigma_{2}$ and $\sigma_{3}$ are selected in accordance with (7.1) the inequalities (4.3) will actually be satisfied or, in other words, that

$$
\frac{\sigma_{22}}{r^{2}} \geqq \sigma_{33} ; \quad \sigma_{33} \geqq \sigma_{11},
$$

for the components $\sigma_{11}, \sigma_{22}$ and $\sigma_{33}$ determined in $\$ 6$.

Substituting the values of the components $\sigma_{11}, \sigma_{22}$ and $\sigma_{33}$ given by (6.16), (6.17) and (6.18) the above inequalities become

$$
\frac{M}{N} \geqq-\frac{1}{3 r^{2}} ; \quad \frac{M}{N} \leqq \frac{1}{3 r^{2}}
$$

Since it was shown in $\S 6$ that $M$ and $N$ are both positive the first inequality (7.2) is automatically satisfied. Now the second inequality (7.2) will be satisfied for all required values of $r$, i.e. for $a \leqq r \leqq b$, if, and only if, we have

$$
\frac{M}{N} \leqq \frac{1}{3 b^{2}}
$$

But, eliminating the ratio $M / N$ by the substitution (6.21), the inequality (7.3) can be written 


$$
\log \frac{b}{a} \leqq \frac{1}{4}\left(\frac{b^{2}}{a^{2}}-\frac{a^{2}}{b^{2}}\right) .
$$

To show that (7.4) is a valid inequality for $b>a$ let us put $x=b / a$ so that the inequality becomes

$$
\log x \leqq \frac{1}{4}\left(x^{2}-\frac{1}{x^{2}}\right), \quad x \geqq 1 .
$$

Now consider the curves $C_{1}$ and $C_{2}$ which are the graphs respectively of the functions appearing in the left and right members of the inequality (7.5). Denote by $\alpha_{1}$ the slope of the curve $C_{1}$ and by $\alpha_{2}$ the slope of the curve $C_{2}$. Thus

$$
\alpha_{1}=\frac{1}{x} ; \quad \alpha_{2}=\frac{1}{2}\left(x+\frac{1}{x^{3}}\right) .
$$

Now the curves $C_{1}$ and $C_{2}$ pass through the same point and have the same slope for $x=1$. Hence if we can show that $\alpha_{1}\left\langle\alpha_{2}\right.$ for $x>1$ it will follow that curve $C_{1}$ lies beneath curve $C_{2}$ for values of $x>1$ or, in other words, that the strict inequality (7.5) is satisfied. But the condition

$$
\frac{1}{x}<\frac{1}{2}\left(x+\frac{1}{x^{3}}\right)
$$

for $x>1$ reduces immediately to the condition $\left(x^{2}-1\right)^{2}>0$ which is automatically satisfied. The principal values $\sigma_{k}$ given by (7.1) therefore satisfy the inequalities (4.3) and hence the Tresca yield condition is furnished by the relation (6.10) as assumed in $\S 6$.

8. Surface of strong instability. Plastic flow. Denote by $\Sigma$ the intersection of the pipe with a half plane through its central axis. It will be shown that $\Sigma$ is a surface of strong instability in the plastically deformed pipe discussed in \$6. Specifically this means that if actual plastic flow is initiated on one side of the plane $\Sigma$ in the plastically deformed pipe the velocity, at points of $\Sigma$, will necessarily become indefinitely large within a finite time. Hence the pipe will be subject to fracture over the plane $\Sigma$ when the internal pressure $p$ has the value given by equation (6.19).

In carrying out the demonstration of this result it will be convenient, although not necessary, to assume that the stress components $\sigma_{i i}$ are continuous over the plane $\Sigma$ at all times. Accordingly this assumption will be made. Thus we can write

$$
\left[\sigma_{i i}\right]=\bar{\sigma}_{i i}-\sigma_{i i}=0,
$$

where the $\sigma_{i j}$ are the stress components defined in $\S 6$ and the $\bar{\sigma}_{i i}$ are the stress components at points on the side of $\Sigma$ facing the region of the plastic flow. From the equations defining the $\sigma_{i j}^{*}$ in $\$ 5$, the conditions (8.1) and the equations for the $\sigma_{i j}$ in $\$ 6$ we now find that 


$$
\left\{\begin{array}{c}
\bar{\sigma}_{11}^{*}=\frac{J}{2}\left(\frac{M r^{2}}{N}-1\right) ; \quad \frac{\bar{\sigma}_{22}^{*}}{r^{2}}=\frac{J}{2}\left(\frac{M r^{2}}{N}+1\right) ; \quad \bar{\sigma}_{33}^{*}=-J \frac{M r^{2}}{N}, \\
\bar{\sigma}_{12}^{*}=0 ; \quad \bar{\sigma}_{13}^{*}=0 ; \quad \bar{\sigma}_{23}^{*}=0,
\end{array}\right.
$$

where the bar, as above, denotes evaluation on the flow side of the plane $\Sigma$. This use of the bar to indicate evaluation on $\Sigma$ of a quantity in the region of plastic flow will be continued in the following discussion.

With regard to the equations for the determination of the plastic flow we assume, in the first instance, that the Tresca yield condition (4.5) is satisfied; however, no explicit use will be made of this full condition. Indeed, it will suffice for our purpose to observe that

$$
\frac{\bar{\sigma}_{22}}{r^{2}}-\bar{\sigma}_{11}=J,
$$

as follows from (6.10) and the above continuity assumption (8.1). In addition we assume the following equations:

$$
\begin{gathered}
g^{i{ }^{i} v_{i, j}}=0, \text { equation of incompressibility, } \\
\boldsymbol{\epsilon}_{i j}=\psi \sigma_{i j}^{*}, \quad \text { von Mises plasticity equations, } \\
g^{i k} \sigma_{i j, k}=\rho\left(\frac{\partial v_{i}}{\partial t}+v_{i, j} v^{i}\right), \text { equations of motion, }
\end{gathered}
$$

in which $\rho$ denotes the density, the $v_{i}$ are the components of velocity and $\psi$ is a positive factor of proportionality. The components $\sigma_{i i}^{*}$ of the stress deviator $\sigma^{*}$ are defined as in $\$ 5$ in terms of the components $\sigma_{i j}$ of the stress tensor $\sigma$. However, the above quantities $\epsilon_{i i}$ are now the components of the rate of strain tensor and are given by

$$
\boldsymbol{\epsilon}_{i j}=\frac{1}{2}\left(v_{i, j}+v_{j, i}\right) .
$$

9. Violation of circular symmetry. The existence of the plane $\Sigma$ separating the regions of plastic equilibrium and plastic flow evidently permits a violation of the condition of circular symmetry used in the foregoing discussion. In other words we are not justified in assuming, from symmetry, that the velocity components $v_{i}$ and the stress components $\sigma_{i j}$ in the region of plastic flow are independent of the angular coordinate $\theta$. However, the stress components $\sigma_{i i}$ and the two velocity components $v_{1}$ and $v_{2}$ can be assumed independent of the longitudinal coordinate $z$ from the symmetry of the problem.

Taking the stress components $\sigma_{i j}$ to be functions

$$
\sigma_{i i}=\sigma_{i j}(r, \theta, t)
$$

in the region of plastic flow let us now calculate the values on the plane $\Sigma$ of certain of the components $\sigma_{i j, k}$ of the covariant derivative of the stress which we shall need in the following discussion. In carrying out this calculation account 
must be taken of the values $\bar{\sigma}_{i i}$ of the stress components which result from the continuity assumption in $\S 8$. But these values are given by the equations

$$
\left\{\begin{array}{l}
\bar{\sigma}_{11}=J \log \frac{r}{b} ; \quad \frac{\bar{\sigma}_{22}}{r^{2}}=J\left(1+\log \frac{r}{b}\right), \\
\bar{\sigma}_{33}=J\left(\log \frac{r}{b}-\frac{3 M}{2 N} r^{2}+\frac{1}{2}\right) ; \quad \bar{\sigma}_{12}=\bar{\sigma}_{13}=\bar{\sigma}_{23}=0,
\end{array}\right.
$$

from which we may infer the above relation (8.3). Hence from (9.1), (9.2) and the formula of covariant differentiation (2.5) we find that

$$
\left\{\begin{array}{lll}
\bar{\sigma}_{11,1}=\frac{\partial \bar{\sigma}_{11}}{\partial r} ; & \bar{\sigma}_{12,2}=\frac{\partial \bar{\sigma}_{12}}{\partial \theta}-J r ; & \bar{\sigma}_{13,3}=0 \\
\bar{\sigma}_{21,1}=0 ; & \bar{\sigma}_{22,2}=\frac{\partial \bar{\sigma}_{22}}{\partial \theta} ; & \bar{\sigma}_{23,3}=0 \\
\bar{\sigma}_{31,1}=0 ; & \bar{\sigma}_{32,2}=0 ; & \bar{\sigma}_{33,3}=0 .
\end{array}\right.
$$

Some simplification can be made in our equations by a suitable restriction on the type of flow and with this end in view the following assumptions will be made. First, the flow takes place in planes $\theta=$ const. It follows from this restriction that $v_{2}=0$. Hence, in particular, $\bar{v}_{2}=0$ which is a necessary requirement in order that there be no immediate separation of material over the plane $\Sigma$. Second, we shall assume that cross sections of the pipe in the region of the plastic flow are displaced into cross sections in this region. The addition of this second restriction obviously implies that the component $v_{3}$ shall be independent of the coordinates $r$ and $\theta$. Hence the components $v_{i}$ will be functions of the form

$$
v_{1}=v_{1}(r, \theta, t) ; \quad v_{2}=0 ; \quad v_{3}=v_{3}(z, t) .
$$

Using the formula (2.4) to calculate the components $v_{i, i}$ of the covariant derivative of the velocity (9.4) we deduce

$$
\left\{\begin{array}{lll}
v_{1,1}=\frac{\partial v_{1}}{\partial r} ; & v_{1,2}=\frac{\partial v_{1}}{\partial \theta} ; & v_{1,3}=0 \\
v_{2,1}=0 ; & v_{2,2}=r v_{1} ; & v_{2,3}=0 \\
v_{3,1}=0 ; & v_{3,2}=0 ; & v_{3,3}=\frac{\partial v_{3}}{\partial z}
\end{array}\right.
$$

Also from (9.5) and the definition of the components $\epsilon_{i j}$ of the rate of strain tensor at the end of $\$ 8$ we have

$$
\left\{\begin{array}{lll}
\epsilon_{11}=\frac{\partial v_{1}}{\partial r} ; & \epsilon_{12}=\frac{1}{2} \frac{\partial v_{1}}{\partial \theta} ; & \epsilon_{13}=0 \\
\epsilon_{22}=r v_{1} ; & \epsilon_{23}=0 ; & \epsilon_{33}=\frac{\partial v_{3}}{\partial z}
\end{array}\right.
$$


Since $\epsilon_{13}$ and $\epsilon_{28}$ are equal to zero it follows immediately from (8.5) that the corresponding stress components $\sigma_{13}$ and $\sigma_{23}$ must also vanish in the region of plastic flow. Hence the quantity $\bar{\sigma}_{32,2}=0$ in (9.3). This fact will be used without further mention in the following discussion.

10. Fracture in pipes. We are now in a position to determine the nature of the plastic flow bordering the plane $\Sigma$. First, expanding the relation (8.4) and using (9.5) we obtain

$$
\frac{\partial v_{1}}{\partial r}+\frac{v_{1}}{r}+\frac{\partial v_{3}}{\partial z}=0
$$

But the third term in this relation depends at most on the variables $z$ and $t$ while the other terms depend only on $r, \theta$ and $t$ from (9.4). Hence we must have

$$
\frac{\partial v_{1}}{\partial r}+\frac{v_{1}}{r}=2 Q(t) ; \quad \frac{\partial v_{3}}{\partial z}=-2 Q(t),
$$

where $Q$ is a function of $t$. Integrating (10.1) we find

$$
\begin{aligned}
& v_{1}=Q(t) r+\frac{S(\theta, t)}{r}, \\
& v_{3}=-2 Q(t) z+R(t),
\end{aligned}
$$

in which $S$ is a function of $\theta$ and $t$ while $R$ depends on $t$ alone.

Now consider the equations (8.5) at points of the plane $\Sigma$. Using (8.2) and (9.6) we see that these equations are satisfied identically for $i, j=1,3$ and $i, j=2,3$. For $i, j=1,2$ we obtain

$$
\frac{\partial \bar{v}_{1}}{\partial \theta}=0 .
$$

Taking $i, j=1,1$ and $i, j=2,2$ in (8.5) leads to the equations

$$
\begin{aligned}
\frac{\partial \bar{v}_{1}}{\partial r} & =\frac{\bar{\psi} J}{2}\left(\frac{M r^{2}}{N}-1\right), \\
\frac{\bar{v}_{1}}{r} & =\frac{\bar{\psi} J}{2}\left(\frac{M r^{2}}{N}+1\right) .
\end{aligned}
$$

Finally if we put $i, j=3,3$ in (8.5) and make use of the second equation (10.1) we find that

$$
\bar{\psi}=\frac{2 Q(t) N}{J M r^{2}}
$$

Let us now eliminate $\bar{\psi}$ from (10.4) and (10.5) by the substitution (10.6). This gives

$$
\frac{\partial \bar{v}_{1}}{\partial r}=Q(t)\left(1-\frac{N}{M r^{2}}\right)
$$




$$
\frac{\bar{v}_{1}}{r}=Q(t)\left(1+\frac{N}{M r^{2}}\right) .
$$

But, evaluating (10.2) on $\Sigma$, the resulting relation must be equivalent to the relation (10.8). This leads to the condition

$$
S(0, t)=\frac{N Q(t)}{M},
$$

where, for definiteness, we have supposed that $\theta=0$ is the equation of the plane $\Sigma$.

It should be observed that (10.7) can be obtained by partial differentiation of (10.8) with respect to $r$. In other words, (10.7) is a consequence of (10.8). Thus we see that, in addition to the equation (10.6) for $\bar{\psi}$, the conditions (8.4) and (8.5) in $\$ 8$ provide us, on the plane $\Sigma$, only with the two independent relations

$$
\frac{\bar{v}_{1}}{r}=Q(t)\left(1+\frac{N}{M r^{2}}\right) ; \quad \bar{v}_{3}=-2 Q(t) z+R(t) .
$$

The first of these is identical with the equation (10.8) and the second is obtained from (10.3) by evaluation on $\Sigma$.

We now consider the equations of motion (8.6) at points on the plane $\Sigma$. Remembering that $\bar{v}_{2}=0$, so that the values of the covariant components $\bar{v}_{i}$ are equal to the values of the corresponding contravariant components $\bar{v}^{i}$, and using the relations (9.3) and (9.5) we find that (8.6) gives

$$
\frac{\partial \bar{\sigma}_{11}}{\partial r}+\frac{1}{r^{2}}\left(\frac{\partial \bar{\sigma}_{12}}{\partial \theta}-J r\right)=\bar{\rho}\left(\frac{\partial \bar{v}_{1}}{\partial t}+\bar{v}_{1} \frac{\partial \bar{v}_{1}}{\partial r}\right),
$$

for $i=1$. Substituting the values of the $\bar{\sigma}_{11}$ and $\bar{v}_{1}$ given by the first equation (9.2) and the first equation (10.9) the above relation (10.10) becomes

$$
\frac{\partial \bar{\sigma}_{12}}{\partial \theta}=\bar{\rho}\left\{\left(r^{3}+\frac{N r}{M}\right) \frac{d Q}{d t}+\left(r^{3}-\frac{N^{2}}{M^{2} r}\right) Q^{2}\right\} .
$$

This is essentially an equation for the determination of the partial derivative of $\sigma_{12}$ with respect to $\theta$ on the plane $\Sigma$. In this connection it might be assumed that $\bar{\rho}$ is constant in view of the condition of incompressibility (8.4) of the plastic material; also the function $Q(t)$ in (10.11) can be assigned the determination found in the following discussion. Similarly, taking $i=2$, the equation (8.6) reduces to

$$
\frac{\partial \bar{\sigma}_{22}}{\partial \theta}=0,
$$

on the plane $\Sigma$. Finally, for $i=3$, we obtain

$$
\frac{\partial \bar{v}_{3}}{\partial t}=2 Q(t) \bar{v}_{3} .
$$


Substituting the value of $\bar{v}_{3}$ given by the second relation (10.9) into the equation (10.12) we obtain the equation

$$
\left(\frac{d Q}{d t}-2 Q^{2}\right) z=\frac{1}{2} \frac{d R}{d t}-Q R .
$$

But, due to the independence of the variables $z$ and $t$, this equation reduces to the following two differential equations for the determination of the functions $Q(t)$ and $R(t)$, namely

$$
\frac{d Q}{d t}=2 Q^{2} ; \quad \frac{d R}{d t}=2 Q R .
$$

Integrating the first of these equations (10.13) and substituting the function $Q(t)$ so obtained into the second equation (10.13), prior to its integration, we find

$$
-2 Q=\frac{1}{t-G} ; \quad R=\frac{H}{t-G},
$$

where $G$ and $H$ are constants independent of the time $t$. In writing the equations (10.14) it is assumed that the plastic flow under consideration is initiated at time $t=0$ so that the admissable values of the time are given by $t \geqq 0$. Hence (10.9) becomes

$$
\bar{v}_{1}=-\frac{M r^{2}+N}{2 M r^{2}(t-G)} ; \quad \bar{v}_{3}=\frac{z+H}{t-G} .
$$

To determine the algebraic sign of the constant $G$ in the equations (10.15) we make use of the fact that $\bar{\psi}>0$ by hypothesis. Hence we must have $Q(t)>0$ from equation (10.6) since the constants $M$ and $N$ were shown to be positive in $\$ 6$. Putting $t=0$ in the first equation (10.14) it therefore follows that

$$
G=\frac{1}{2 Q(0)}>0
$$

Allowing the time $t$ to increase from its initial value $t=0$ to the value $t=G$ $(>0)$ it is seen immediately from the equations (10.15) that the values of the quantities $\bar{v}_{1}$ and $\bar{v}_{3}$ will approach infinity. Hence $\Sigma$ is a surface of strong instability and the pipe is therefore subject to fracture over this surface in accordance with the criterion stated in $\$ 1$. As previously mentioned the equation (6.19) will give the internal pressure $p$ at which such fracture will occur.

Remark. For unloading at the yield point ${ }^{6}$ we have $\bar{\psi}<0$. But $\bar{\psi}<0$ implies $G<0$ by a consideration similar to that used in obtaining the condition (10.16). From the equations (10.15) we can therefore infer the following result. If unloading at the yield point takes place the velocity $\bar{v}$ will be damped out and hence fracture will not occur over the plane $\Sigma$.

6 See W. Prager \& P. G. Hodge, Jr., Theory of Perfectly Plastic Solids, J. Wiley and Sons, New York, 1951, p. 29. 
Addendum. It has been pointed out to me by Tsuan Wu Ting that the use of the strong condition $v_{2}=0$ in the region of plastic flow contradicts the assumption that not all velocity components $v$ vanish on the surface $\Sigma$, i.e. the plane $\theta=0$, provided we postulate the existence of derivatives of higher order than those used in the above discussion; this contradiction occurs as a consequence of conditions which are obtained by differentiation of the basic equations governing the plastic flow. The difficulty can be avoided, however, if we replace the condition $v_{2}=0$ by the following boundary conditions, namely

$$
\bar{v}_{2}=0 ; \quad \frac{\partial \bar{v}_{2}}{\partial \theta}=0
$$

which suffice, as is easily seen, for the demonstration of the above results. The first of these boundary conditions gives the necessary requirement that there is no immediate separation of material over the surface $\Sigma$, as stated above, while the second condition can be expressed by saying that the flow remains in a plane $\theta=$ const. to the first approximation in the immediate neighborhood of the surface $\Sigma$.

Graduate Institute for Mathematics and Mechanics

Indiana University 